\title{
Participación del sistema endocanabinoide en el desarrollo de obesidad
}

\author{
CARINA VALENZUELA ${ }^{\mathrm{a}}$, CAROLINA AGUIRRE ${ }^{\mathrm{a}}$, VALESKA CASTILLO ${ }^{\mathrm{b}}$, \\ ANA MARÍA RONCO ${ }^{b}$, MIGUEL LLANOS
}

\section{A role for the endocannabinoid system in obesity}

Endocannabinoids are the endogenous ligands for the cannabinoid receptors type 1 and 2. These membrane receptors are responsible for the psychotropic effects of Cannabis Sativa, when bound to its active component known as (-)- $\Delta^{9}$-tetrahydrocannabinol. Cannabinoid receptors, endocannabinoids and the enzymes catalyzing their biosynthesis and degradation, constitute the endocannabinoid system (ECS), which has a remarkable role controlling energy balance, both at central nervous system and peripheral tissues. The ECS regulates food ingestion by stimulating a network of orexigenic neurons present in the hypothalamus and reinforcing motivation and reward to food consumption in the nucleus accumbens. Regarding peripheral tissues, this system controls lipid and glucose metabolism at different levels, reduces energy expenditure and leads energy balance to fat storage. Metabolic alterations, including excessive accumulation of abdominal fat, dyslipidaemia and hyperglicaemia, are suggested to be associated to a hyperactivated ECS. Since obesity is one of the major health problems in modern societies, in this review we discuss the role of the endocannabinoid system in metabolic pathways associated to control mechanisms of energy balance and its involvement in overweight and obesity. In addition, we also discuss therapeutic possibilities and emergent problems due to cannabinoid receptor type 1 antagonism utilized as treatment for such alterations.

(Rev Med Chile 2010; 138: 621-629).

Key words: $C B_{1}$ Receptor; Endocannabinoids; Lipogenesis; Obesity.

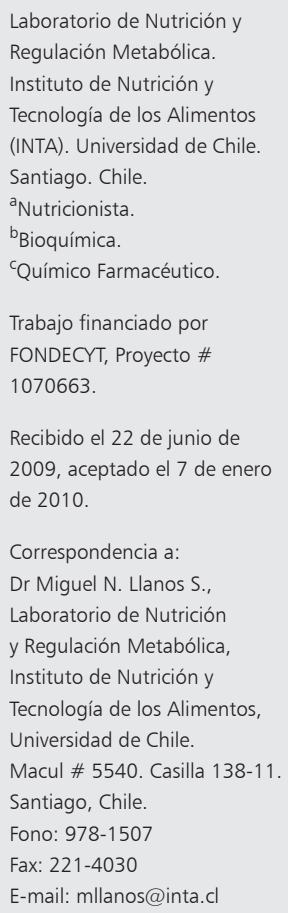

L a homeostasis de energía está regulada por complejos mecanismos centrales y periféricos interconectados entre si, los cuales interactúan con el fin de mantener niveles adecuados de ingesta, almacenamiento y utilización de la energía. Los factores ambientales asociados al estilo de vida actual han llevado a una aparente desregulación de estos mecanismos y por lo tanto a una elevada incidencia de obesidad, la cual se asocia con un aumento del riesgo de insulino resistencia, diabetes tipo 2, hígado graso no alcohólico, dislipidemia no aterogénica y enfermedades cardiovasculares. Actualmente, el sistema endocanabinoide (SEC) es reconocido como un sistema clave en aspectos relacionados con la homeostasis de energía ya que tiene un rol en la ingesta alimentaria, la lipogénesis hepática, la adipogénesis y lipogénesis del tejido adiposo, así como en el metabolismo de la glucosa. En esta revisión se discuten los hallazgos que relacionan al SEC con la etiología de la obesidad y los mecanismos involucrados, además de sus implicancias terapéuticas.

\section{Características generales del sistema endocanabinoide}

El SEC lo comprenden dos receptores para canabinoides: $\mathrm{RCB}_{1} \mathrm{y}\left(\mathrm{RCB}_{2}\right)^{1}$, sus ligandos endó- 
genos denominados endocanabinoides, siendo los más estudiados la anandamida (ADA) y el 2-araquidonilglicerol $(2-\mathrm{AG})^{2}$, y las enzimas responsables de su formación y degradación.

Los $\mathrm{RCB}_{1}$ y $\mathrm{RCB}_{2}$ son receptores de membrana acoplados a proteína $\mathrm{G}$, que fueron descubiertos como blancos moleculares del componente psicotrópico de la planta Cannabis Sativa, el (-)- $\Delta^{9}$-Tetrahidrocannabinol $\left(\Delta^{9}\right.$-THC), y participan en la modulación fisiológica de muchas funciones centrales y periféricas. El $\mathrm{RCB}_{2}$ se expresa principalmente en las células del sistema inmune, mientras que el $\mathrm{RCB}_{1}$ se expresa tanto a nivel central como periférico ${ }^{3-7}$. Ambos receptores pueden ser antagonizados selectivamente, siendo los más estudiados el compuesto SR141716A, que antagoniza al $\mathrm{RCB}_{1}$, y el compuesto SR144528 que antagoniza al $\mathrm{RCB}_{2}^{8-10}$.

Los endocanabinoides ADA y 2-AG son biosintetizados a demanda, a partir de fosfolípidos de membrana por la acción de distintas fosfolipasas y, posterior a su liberación, son degradados enzimáticamente. La anandamida es degradada específicamente por la enzima ácido graso ami- do hidrolasa (FAAH), mientras que el 2-AG es inactivado por la enzima monoacilglicerol lipasa. Las vías de síntesis y degradación se han descrito fundamentalmente a nivel neuronal, pero es muy probable que sean similares en otros tejidos (Figura 1).

\section{Endocanabinoides y regulación de la ingesta de alimentos}

La regulación de la ingesta de alimentos por el SEC ocurre a nivel del sistema nervioso central. De hecho, los efectos antieméticos e inductores del apetito del $\Delta^{9}$-THC, son conocidos desde hace bastante tiempo y por esta razón este compuesto, así como la nabilona, su análogo de síntesis, se han utilizado para disminuir los vómitos y náuseas en pacientes con cáncer, y para prevenir la pérdida de peso en pacientes con SIDA ${ }^{7}$. El aumento de la ingesta por acción del $\Delta^{9}$-THC y de los endocanabinoides ${ }^{11-13}$ se produce a través de la estimulación de $\mathrm{RCB}_{1}$, ya que la inhibición de éstos por la administración de SR141716A,

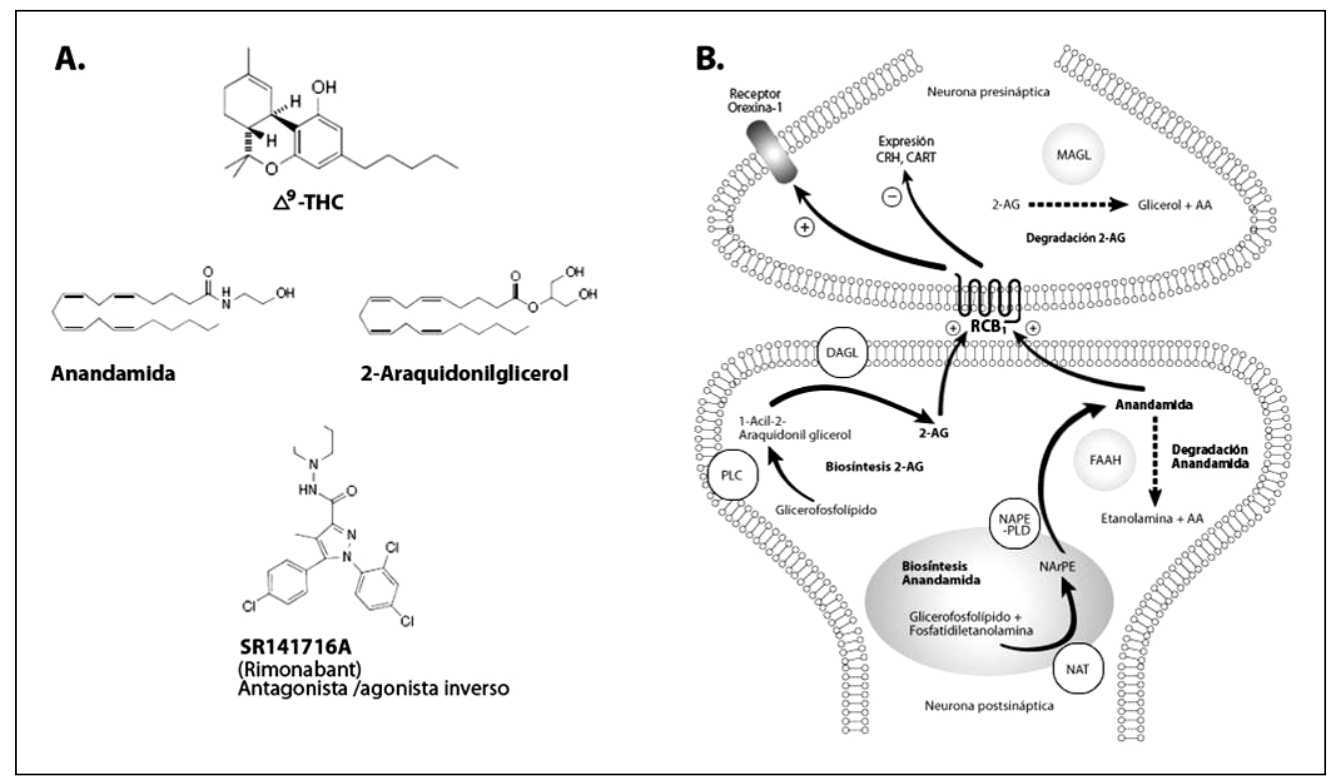

Figura 1. A) Estructura química de los principales agonistas y del antagonista clásico del RCB. B) Vías de síntesis, degradación de endocanabinoides y su acción retrograda en el RCB, pre-sináptico. AA: ácido araquidónico; FAAH: ácido graso amido hidrolasa; PLC: fosfolipasa C; MAGL: monoacilglicerol lipasa; DAGL: diacilglicerol lipasa; NAPE-PLD: Fosfolipasa D selectiva para $\mathrm{N}$-acilfosfatidil-etanolamida; NAT: N-aciltransferasa; 2-AG: 2-araquidonilglicerol; NarPE: N-araquidonil-fosfatidil-etanolamida; $\mathrm{CRH}$ : hormona liberadora de corticotrofina; CART: transcripto regulado por cocaína y anfetamina. 
antagonista/agonista inverso de $\mathrm{RCB}_{1}$, reduce el consumo de alimentos ${ }^{11,14}$. El RCB $\mathrm{RC}_{1}$ se expresa en áreas del hipotálamo involucradas en el control del apetito, como es el núcleo paraventricular (NPV) y el hipotálamo lateral (HL). Los endocanabinoides hipotalámicos liberados por las neuronas postsinápticas participan en el control de la ingesta alimentaria regulando la expresión y/o acción de varios mediadores anorexígenos y orexígenos ${ }^{15}$, a través un mecanismo de regulación retrógrada mediado por $\mathrm{RCB}_{1}$, localizados mayoritariamente a nivel pre-sináptico ${ }^{16,17}$ (Figura 1). En el NPV, la estimulación de $\mathrm{RCB}_{1}$ reduce la liberación de la hormona liberadora de corticotrofinas $(\mathrm{CRH})$ un compuesto de carácter anorexígeno ${ }^{18}$. En el HL, los endocanabinoides estimulan la excitabilidad de las neuronas que contienen la hormona concentradora de melanina $(\mathrm{MCH})^{19}$, generando un aumento de la ingesta. Mientras que la activación de $\mathrm{RCB}_{1}$ inhibe la liberación del transcripto anorexígeno relacionado a cocaína y anfetamina $(\mathrm{CART})^{20}$. Adicionalmente el $\mathrm{RCB}$ coexiste con los receptores serotonérgicos 5-HT1B y 5-HT3 en la formación hipocampal y en la neocorteza ${ }^{21}$. De manera que la interacción SEC-sistema serotoninérgico podría dar cuenta de otra vía involucrada en el control de la ingesta.

Se ha sugerido que el SEC tiene un rol importante en la modulación del circuito de recompensa y reforzamiento de la ingesta, ya que $R B_{1}$ se co-localiza con los receptores dopaminérgicos D1 y D2 en el sistema mesolímbico ${ }^{21}$. Así, la "conversación-cruzada" entre ambos sistemas se ha demostrado en experimentos en los cuales antagonistas del receptor D1 reducen el estímulo orexigénico inducido por $\Delta^{9}-\mathrm{THC}^{22}$. Por otro lado, el bloqueo de $\mathrm{RCB}_{1}$ por SR141716A reduce el aumento de dopamina estimulado por el consumo de alimentos palatables ${ }^{23}$. También se ha postulado una interacción funcional entre los sistemas endocanabinoide y opioide en los mecanismos de recompensa a nivel central ${ }^{22}$.

Es interesante el hecho que los niveles hipotalámicos de endocanabinoides están inversamente correlacionados con los niveles sanguíneos de la hormona leptina; así, se sabe que ratones obesos con señalización defectuosa de leptina (ratones $\mathrm{ob} / \mathrm{ob}$ y db/db) muestran niveles elevados de endocanabinoides (ADA y 2-AG) hipotalámicos ${ }^{15}$, y que la administración de leptina disminuye concomitantemente la ingesta de alimentos y los niveles de ADA y 2-AG en ratones normales y ob/ ob. Por lo tanto, los efectos cruciales de leptina en la regulación de mediadores orexígenos y anorexígenos hipotalámicos implican la participación de endocanabinoides y $\mathrm{RCB}_{1}$, los que parecen estar involucrados en la hiperfagia y obesidad en situaciones de señalización defectuosa de leptina ${ }^{15}$.

\section{Endocanabinoides y regulación del metabolismo energético}

El bloqueo de $\mathrm{RCB}_{1}$ por SR141716A reduce la ingesta de alimentos en modelos genéticos de obesidad tales como ratones ob/ob, db/db y ratas Zucker ${ }^{24}$. Sin embargo, el efecto de los bloqueadores de $\mathrm{RCB}_{1}$ sobre la ingesta es transitorio, mientras que la disminución de peso es sostenida en el tiempo ${ }^{24}$, lo que se observó en estudios con alimentación pareada y en modelos animales de obesidad inducida por dieta. En estos últimos, el tratamiento crónico con bloqueador del $\mathrm{RCB}_{1}$ produce una reducción significativa de la masa grasa en relación a la magra, y una mejora de ciertos parámetros metabólicos, como niveles de glucosa, insulina, leptina y colesterol, entre otros ${ }^{25,26}$. Notablemente, los ratones knockout para $\mathrm{RCB}_{1}$ $\left(\mathrm{RCB}_{1}^{-/-}\right)$, presentan un fenotipo magro, además de una tendencia a un mayor gasto energético en la fase oscura, que los ratones nativos con alimentación pareada ${ }^{27}$. Incluso con una dieta alta en grasa, los ratones $\mathrm{RCB}_{1}^{-/-}$no desarrollan obesidad o insulino-resistencia, a pesar de que consumen tanto alimento como sus pares nativos ${ }^{28}$. Estos antecedentes demuestran que la reducción de peso y masa grasa, producto del tratamiento con antagonistas $\mathrm{RCB}_{1}$, se deben inicialmente a su acción anorexígena a nivel central y posteriormente a un efecto a nivel periférico, sugiriendo la participación del SEC en la regulación periférica del metabolismo energético, lo que ha generado estudios asociados a lipogénesis, primeramente en tejido graso ${ }^{27}$ y luego en hígado ${ }^{4}$, entre otros.

Diversos hallazgos sugieren que el incremento de la actividad del SEC contribuye a la acumulación de triglicéridos en el tejido adiposo, independiente de la cantidad de alimento consumido. En este sentido, se ha observado la expresión de $\mathrm{RCB}_{1}$ en grasa epididimal de ratones machos y en cultivos primarios de adipocitos. La estimulación de dichos adipocitos con WIN-55,212, agonista 
de $\mathrm{RCB}_{1}$, aumenta en forma dosis-dependiente la actividad de la lipasa lipoproteica, efecto que es bloqueado por SR141716A, demostrando que este efecto es mediado por $\mathrm{RCB}_{1}{ }^{27}$. Otro aspecto importante de señalar es la mayor expresión de $\mathrm{RCB}_{1}$ en ratas obesas Zucker (fa/fa), al compararlas con ratas control ${ }^{5}$. El bloqueo de RCB en estas ratas es capaz de estimular la expresión de adiponectina, hormona sintetizada en tejido adiposo que promueve la oxidación muscular de ácidos grasos y la reducción de peso ${ }^{29}$. Otros estudios demuestran además que SR141716A disminuye la hiperglicemia, la hiperinsulinemia y la acumulación de grasa visceral en modelos animales de estrés, diabetes y obesidad ${ }^{30-32}$.

Dado que el rol del tejido adiposo en lipogénesis de novo es menor en comparación con dicha función a nivel hepático ${ }^{33}$, diversos estudios han apuntado a examinar al hígado y su maquinaria molecular como un tejido blanco para las acciones metabólicas de los endocanabinoides. Se ha observado la expresión de $\mathrm{RCB}_{1}$ en hepatocitos, cuya activación estimula la expresión de un importante factor de transcripción lipogénico, la proteína de unión del elemento regulatorio de esteroles 1c (SREBP-1c) y de sus genes blanco, las enzimas acetil-CoA-carboxilasa-1 (ACC1) y la sintetasa de ácidos grasos (FAS $)^{4}$. Esto probablemente explica los efectos de agonistas para $\mathrm{RCB}_{1}$ en la estimulación de la síntesis de ácidos grasos de novo y lipogénesis en células hepáticas ${ }^{4}$. La ausencia de respuesta lipogénica hepática en ratones $\mathrm{RCB}_{1}{ }^{-1} \mathrm{y}$ la inhibición de ésta en ratones nativos por acción del antagonista del RCB 1 , SR141716A, dan mayor respaldo a la función lipogénica de $\mathrm{RCB}$ hepáticos. Este mecanismo parece contribuir a la obesidad inducida por dieta, la que está asociada a niveles hepáticos elevados de $\mathrm{RCB}_{1} \mathrm{y}$ de $\mathrm{ADA}$, al parecer por una disminución en la actividad de la enzima FAAH. De esta forma, la estimulación sostenida de $\mathrm{RCB}_{1}$ generaría síntesis de ácidos grasos de novo en hígado de ratón, la cual no se observa en hígado ni en hepatocitos aislados de ratones tratados con SR141716A ni en ratones $\mathrm{RCB}_{1}^{-1-4}$. Estudios más recientes indican que ratones knockout $\mathrm{RCB}_{1}$ hígado-específico alimentados con una dieta alta en grasa, presentan menos esteatosis, hiperglicemia, dislipidemia y resistencia a insulina y leptina, al compararlos con los ratones nativos alimentados con la misma dieta hipergrasa. Estos resultados indican que la activación de $\mathrm{RCB}_{1}$ hepáticos con- tribuye al desarrollo de esteatosis inducida por la dieta y a las alteraciones hormonales y metabólicas asociadas $^{34}$. De esta forma, es posible que factores hepáticos liberados a la circulación y dependientes de la hiperactivación de $\mathrm{RCB}_{1}$, estén asociados al desencadenamiento de ciertas condiciones fisiopatológicas ${ }^{35}$.

Otros hallazgos sugieren que los endocanabinoides están involucrados en el control del metabolismo de hidratos de carbono por medio de la regulación de los niveles de insulina, así como de la captación y utilización de glucosa por los tejidos, con el posterior impacto en los niveles de glucosa circulante. $\mathrm{Al}$ respecto, se ha demostrado la expresión de $\mathrm{RCB}_{1}$ y $\mathrm{RCB}_{2}$ en páncreas endocrino, los primeros en células $\alpha$ y los segundos tanto en células $\alpha$ como en $\beta^{36}$. Además, en insulinoma de ratas se ha observado que la producción de endocanabinoides está regulada negativamente por insulina ${ }^{32}$ y existe evidencia que indica que la hiperactivación del SEC durante períodos de hiperglicemia podría contribuir a la hiperinsulinemia característica de la obesidad ${ }^{36}$. Por otro lado, se ha observado la expresión de $\mathrm{RCB}_{1}$ en músculo esquelético humano y de ratones $^{137,38}$. En este sentido, ratones $\mathrm{RCB}_{1}^{-/-}$muestran una tendencia hacia un mayor gasto energético ${ }^{27}$, mientras que un tratamiento de 7 días con SR141716A aumenta en $37 \%$ el consumo de oxígeno (activación de termogénesis) en ratones genéticamente obesos (ob/ob), produciendo, además, un incremento significativo de la captación de glucosa en músculo soleo $^{3}$. Se ha observado también que la expresión de $\mathrm{RCB}_{1}$ en músculo soleo de ratón, aumenta al someterlos a una dieta alta en grasa ${ }^{38}$.

\section{Perturbaciones del sistema endocanabinoide en obesidad}

Los $\mathrm{RCB}_{1}$ podrían jugar un rol crucial en la regulación del gasto energético en humanos. Esto se observó con el uso de un antagonista $\mathrm{RCB}_{1}$ en sujetos con sobrepeso y obesos, donde el gasto energético basal aumentó significativamente con respecto al grupo placebo, luego de 2 a 5 h posttratamiento ${ }^{39}$. Dada la secreción local y rápida degradación de los endocanabinoides, la importancia de los niveles circulantes de estos compuestos no es totalmente comprendida, ya que podrían reflejar el recambio de células sanguíneas circulantes o los 
endocanabinoides liberados por órganos periféricos. Sin embargo, varios estudios han encontrado diferencias entre distintos subgrupos de pacientes. Por ejemplo, los niveles circulantes de ADA y $2-A G$ están aumentados en mujeres post-menopáusicas obesas, en comparación con aquellas mujeres postmenopáusicas con mayor masa magra ${ }^{6}$, mientras que mujeres obesas con trastornos alimentarios ("binge eating disorder") presentan niveles plasmáticos de ADA 2 veces mayores que sus controles ${ }^{40}$. Estos hallazgos sugieren un aumento periférico del tono endocanabinoide, además del posible rol de ADA en aspectos hedónicos de algunos desórdenes alimentarios ${ }^{40}$.

Por otra parte, el aumento de niveles de 2-AG solamente se ha observado en tejido adiposo visceral, pero no subcutáneo, en humanos con sobrepeso y obesos ${ }^{32}$. La hiperactivación del SEC en los depósitos adiposos visceral vs subcutáneo fue confirmada por estudios que mostraron una correlación positiva entre los niveles plasmáticos de 2-AG, pero no de ADA, y la cantidad de grasa intra-abdominal ${ }^{41,42}$. En pacientes diabéticos tipo 2 no obesos (IMC $\approx 30$ ) y con hiperglicemia parcialmente controlada, los niveles plasmáticos de ADA y 2-AG están aumentados al compararlos con sujetos controles no diabéticos ${ }^{32}$. Una posible causa del aumento de los niveles de endocanabinoides es una menor expresión de sus enzimas de degradación, lo que se ha observado en grasa visceral y subcutánea de pacientes obesos $s^{6,41,43}$.

La relación entre un SEC disfuncional y obesidad podría involucrar también polimorfismos genéticos. Así, se ha observado una correlación entre sobrepeso/obesidad y un polimorfismo en el gen $\mathrm{FAAH}^{44}$, que genera una enzima con menor actividad degradatoria de ADA.

Los resultados en relación a la expresión de $\mathrm{RCB}_{1}$ en sujetos obesos también son contradictorios. Se ha observado una menor expresión en tejido adiposo omental y subcutáneo ${ }^{6,41,45}$. Mientras que en un estudio realizado en pacientes obesos no se encontró cambio en los niveles de ARNm para $\mathrm{RCB}_{1}$ en ningún tipo de depósito graso corporal ${ }^{43}$. También se ha reportado que los niveles de ARNm para $\mathrm{RCB}_{1}$ están aumentados en la grasa subcutánea y visceral, y disminuidos en la grasa subcutánea glútea ${ }^{46}$. Asimismo, se han encontrado correlaciones entre polimorfismos en CNR1, el gen que codifica para el $\mathrm{RCB}_{1}$, y la ocurrencia de fenotipos magros u obesos en varias poblaciones humanas adultas ${ }^{47,48}$. La relevancia patológica de estos hallazgos debe esperar más estudios que investiguen el impacto de estas mutaciones en la actividad funcional del $\mathrm{RCB}_{1}$.

Adicionalmente, una desregulación de los niveles de endocanabinoides puede ocurrir por acciones alteradas de leptina e insulina. En obesidad, la leptina pierde su capacidad para inhibir la ingesta energética y aumentar el gasto energético ${ }^{49}$, lo que podría explicar el impacto negativo de una señalización defectuosa de leptina en el cerebro ${ }^{50}$. Esta situación también podría aumentar los niveles de endocanabinoides en el hipotálamo, con la posterior hiperfagia y aumento de peso corporal. Por otro lado, la insulina genera una disminución de los niveles de endocanabinoides en insulinoma de células $\beta$, cultivadas con niveles bajos de glucosa $a^{32} y$ estimula la expresión de la enzima FAAH en tejido adiposo subcutáneo de sujetos magros, pero no de aquellos obesos ${ }^{51}$, donde los altos niveles de 2-AG se correlacionan con bajos niveles de ARNm para FAAH en tejido adiposo visceral ${ }^{41}$. Por lo tanto, niveles aumentados de endocanabinoides también podrían generarse por insulino resistencia, fundamentalmente en tejidos periféricos.

La Figura 2 resume los efectos de un SEC hiperactivo en diferentes tejidos que se traducen en último término en el desarrollo de sobrepeso/ obesidad y sus alteraciones metabólicas asociadas.

\section{Problemas emergentes asociados a tratamientos con antagonista $\mathrm{RCB}$}

Los resultados obtenidos en los diferentes programas RIO ("Rimonabant in Obesity/ Overweight"), demostraron que el bloqueo crónico de los RCB con el compuesto Rimonabant en individuos obesos con síndrome metabólico generó pérdida de peso y un considerable mejoramiento en el perfil lipídico de estos pacientes, acompañado además de un aumento en la sensibilidad a la insulina ${ }^{52,53}$. Estos resultados, claramente positivos, llevaron a la aprobación en Europa (no así en Estados Unidos de Norteamérica) de la utilización masiva del compuesto Rimonabant durante alrededor de dos años, período en el cual fue posible constatar que la mayor limitante de este compuesto era el bloqueo no selectivo de $\mathrm{RCB}_{1}$ en el sistema nervioso central, hecho que eventualmente llevó a problemas de ansiedad y 


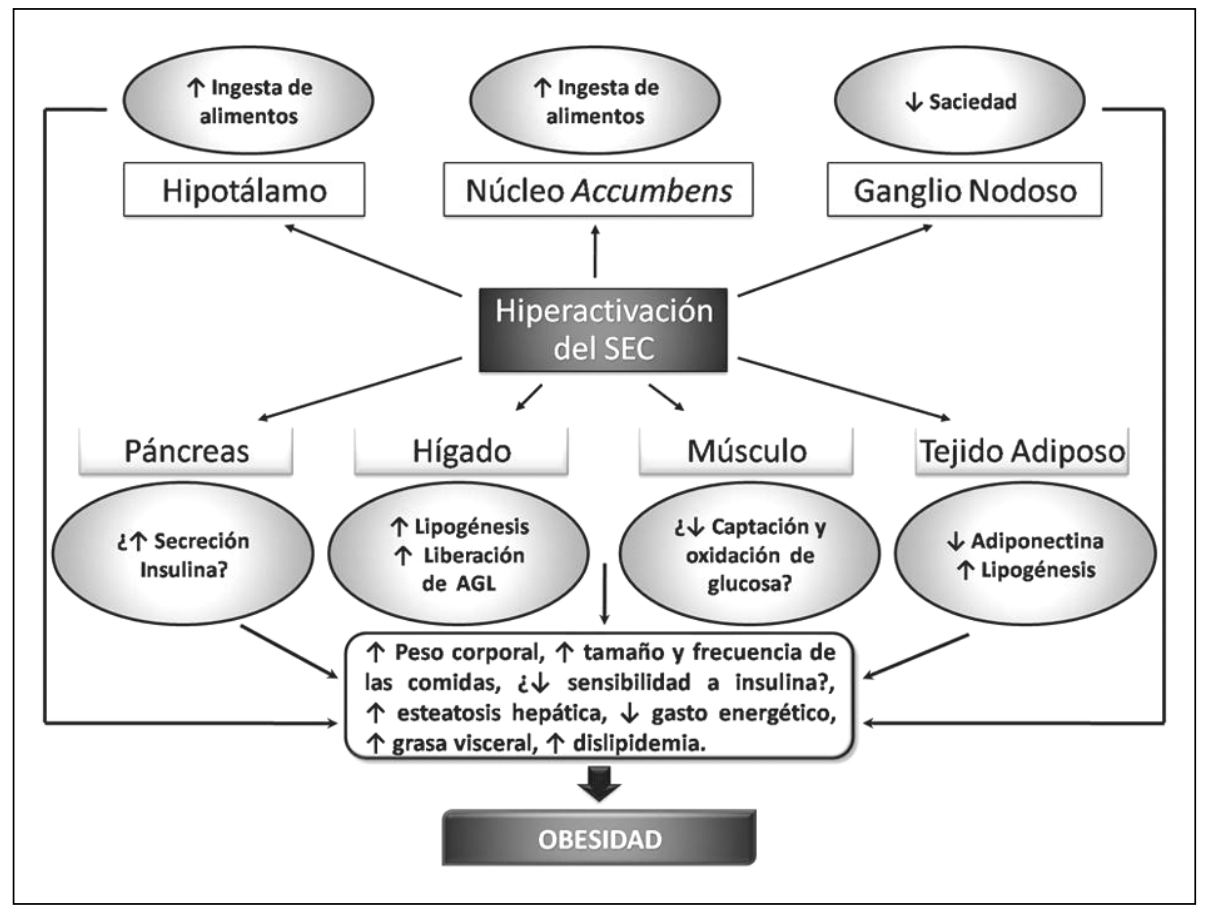

Figura 2. Consecuencias de un sistema endocanabinoide hiperactivo a nivel del sistema nervioso central y tejidos periféricos, mediadas por aumento o sobre-estimulación de RBC. AGL: ácidos grasos libres. depresión marcada en pacientes susceptibles ${ }^{54}$. Es necesario recordar que el SEC está asociado a la mantención de un estado homeostático de comportamiento, respondiendo a períodos de estrés y ansiedad a través de la liberación protectora de $\mathrm{ADA}$ y $2-\mathrm{AG}^{18}$, que actúan sobre $\mathrm{RCB}_{1}$ para generar una modulación neuronal retrógrada ${ }^{55,56}$. De manera que el bloqueo de $\mathrm{RCB}_{1}$ en el sistema nervioso central debiese afectar los mecanismos de "manejo" del estrés y la ansiedad, facilitando la manifestación de efectos secundarios con carácter depresivo. Dado que fue posible constatar que los riesgos de desórdenes siquiátricos a causa de este compuesto sobrepasaban los efectos beneficiosos, la Agencia Europea de Medicina recomendó en octubre de 2008 la suspensión de la venta de esta droga para el tratamiento de la obesidad ${ }^{57}$. La empresa Sanofi-Aventis siguió estas recomendaciones y determinó finalizar todos los estudios clínicos en desarrollo con este compuesto. A esto se ha sumado recientemente la detención de otros estudios clínicos ya en Fase III, que utilizaban los bloqueadores de $\mathrm{RCB}_{1}$ taranabant (Merck) y CP945,598 (Pfizer) como agentes antiobesidad. Estos antecedentes sugieren que indicar al $\mathrm{RCB}_{1}$ como un blanco para el tratamiento de la obesidad ${ }^{57}$ ya no sería un paradigma vigente, dado que este no tendría un sustento práctico de aplicación terapéutica beneficiosa para el ser humano. Sin embargo, es posible que el abandono de esta posibilidad terapéutica sea prematuro, ya que los resultados obtenidos durante dos años en el estudio RIOEuropa indican otros efectos susceptibles de ser aprovechados. Adicionalmente, la posibilidad que la investigación actual sea reorientada hacia la obtención de bloqueadores $\mathrm{RCB}_{1}$ que sean incapaces de atravesar la barrera hematoencefálica también es plausible. De esta manera, el blanco natural de dichos compuestos serían los $\mathrm{RCB}_{1}$ que se encuentran a nivel periférico en los diversos tejidos ya descritos. Al respecto, estudios recientes en animales indican que los efectos metabólicos beneficiosos están mediados preferentemente por los $\mathrm{RCB}_{1}$ presentes en tejidos periféricos ${ }^{34,58}$ y que, por lo tanto, antagonistas de $\mathrm{RCB}$ restringidos a la periferia deberían retener su eficacia terapéutica reduciendo los potenciales efectos colaterales a nivel del sistema nervioso central ${ }^{35}$. En efecto, un estudio preliminar reciente describe que un tratamiento oral con un antagonista restringido sólo a $\mathrm{RCB}_{1}$ periféricos es efectivo en el manejo de la obesidad y sus alteraciones metabólicas 
asociadas $^{59}$. En conclusión y pese a las recientes decepciones terapéuticas en este campo de investigación, la manipulación del SEC sigue siendo una estrategia prometedora para el tratamiento de la obesidad y sus comorbilidades.

\section{Referencias}

1. Devane WA, Dysarz FA 3rd, Johnson MR, Melvin LS, Howlett AC. Determination and characterization of a cannabinoid receptor in rat brain. Mol Pharmacol 1988; 34: 605-13.

2. Devane WA, Hanus L, Breuer A, Pertwee RG, Stevenson LA, Griffin $G$, et al. Isolation and structure of a brain constituent that binds to the cannabinoid receptors. Science 1992; 258: 1946-9.

3. Liu YL, Connoley IP, Wilson CA, Stock MJ. Effects of the cannabinoid CB1 receptor antagonist SR141716 on oxygen consumption and soleus muscle glucose uptake in Lep(ob)/Lep(ob) mice. Int J Obes 2005; 29: 183-7.

4. Osei-Hyiaman D, DePetrillo M, Pacher P, Liu J, Radaeva S, Batkai S, et al. Endocannabinoid activation at hepatic CB1 receptors stimulates fatty acid synthesis and contributes to diet-induced obesity. J Clin Invest 2005; 115: 1298-305.

5. Bensaid M, Gary-Bobo M, Esclangon A, Maffrand JP, Le Fur G, Oury-Donat F, et al. The cannabinoid CB1 receptor antagonist SR141716 increased Acrp30 mRNA expression in adiopose tissue of obese fa/fa rats and culture adipocyte cells. Mol Pharmacol 2003; 63: 908-914.

6. Engeli S, Böhnke J, Feldpausch M, Gorzelniak K, Janke J, Bátkai S, et al. Activation of the peripheral endocannabinoid system in human obesity. Diabetes 2005; 54: 2838-43.

7. Matias I, Di Marzo V. Endocannabinoids and the control of energy balance. Trends Endocrinol Metab 2007; 18: 27-37.

8. Pertwee, RG. Inverse agonism and neutral antagonism at cannabinoid $\mathrm{CB}_{1}$ receptors. Life Sci 2005; 76: 1307-24.

9. Piomelli D, Giuffrida A, Calignano A, Rodríguez de Fonseca $\mathrm{F}$. The endocannabinoid system as a target for therapeutic drugs. Trends Pharmacol Sci 2000; 21: 21824.

10. Ronco AM, Llanos M, Tamayo D, Hirsch S. Anandamide inhibits endothelin-1 production by human culture endothelial cells: a new vascular action of this endocannabinoid. Pharmacology 2007; 79: 12-6.

11. Williams CM, Rogers PJ, Kirkham TC. Hyperphagia in pre-fed rats following oral delta 9-THC. Physiol Behav 1998; 65: 343-6.

12. Kirkham TC, Williams CM, Fezza F, Di Marzo V. En- docannabinoid levels in rat limbic forebrain and hypothalamus in relation to fasting, feeding and satiation: stimulation of eating by 2-arachidonoyl glycerol. Br J Pharmacol 2002; 136: 550-7.

13. Jamshidi N, Taylor DA. Anandamide administration into the ventromedial hypothalamus stimulates appetite in rats. Br J Pharmacol 2001; 134: 1151-4.

14. Colombo G, Agabio R, Díaz G, Lobina C, Reali R, Gessa GL. Appetite suppression and weight loss after the cannabinoid antagonist SR 141716. Life Sci 1998; 63: PL113-17.

15. Di Marzo V, Goparaju SK, Wang L, Liu J, Bátkai S, Járai $Z$, et al. Leptin-regulated endocannabinoids are involved in maintaining food intake. Nature 2001; 410: 822-5.

16. Horvath, TL. Endocannabinoids and the regulation of body fat: the smoke is clearing. J Clin Inv 2003; 112: 323-6.

17. Wilson RI, Nicoll RA. Endocannabinoid signaling in the brain. Science 2002; 296: 678-82.

18. Di S, Malcher-Lopes R, Halmos KC, Tasker JG. Nongenomic glucocorticoid inhibition via endocannabinoid release in the hypothalamus: a fast feedback mechanism. J Neurosci 2003; 23: 4850-7.

19. Jo YH, Chen YJ, Chua SC Jr, Talmage DA, Role LW. Integration of endocannabinoid and leptin signaling in an appetite-related neural circuit. Neuron. 2005; 48(6): 1055-66.

20. Osei-Hyiaman D, Depetrillo M, Harvey-White J, Bannon AW, Cravatt BF, Kuhar MJ, et al. Cocaine- and amphetamine-related transcript is involved in the orexigenic effect of endogenous anandamide. Neuroendocrinology 2005; 81: 273-82.

21. Hermann H, Marsicano G, Lutz B. Coexpression of the cannabinoid receptor type 1 with dopamine and serotonin receptors in distinct neuronal subpopulations of the adult mouse forebrain. Neuroscience 2002; 109: 451-60.

22. Bellocchio L, Cervino C, Pasquali R, Pagotto U. The endocannabinoid system and energy metabolism. J Neuroendocrinol 2008; 20: 850-857.

23. Melis T, Succu S, Sanna F, Boi A, Argiolas A, Melis MR. The cannabinoid antagonist SR141716A (Rimonabant) reduces the increase of extra-cellular dopamine release in the rat nucleus accumbens induced by a novel high palatable food. Neurosc Lett 2007; 419: 231-5.

24. Vickers SP, Webster LJ, Wyatt A, Dourish CT, Kennett GA. Preferential effects of the cannabinoid CB1 receptor antagonist, SR 141716, on food intake and body weight gain of obese ( $\mathrm{fa} / \mathrm{fa}$ ) compared to lean Zucker rats. Psychopharmacology (Berl) 2003; 167: 103-11.

25. Ravinet Trillou C, Arnone M, Delgorge C, Gonalons N, Keane P, Maffrand JP, et al. Anti-obesity effect of 
Participación del sistema endocanabinoide en el desarrollo de obesidad - C. Valenzuela et al

SR141716, a CB1 receptor antagonist, in diet-induced obese mice. Am J Physiol Regul Integr Comp Physiol 2003; 284: R343-4.

26. Poirier B, Bidouard JP, Cadrouvele C, Marniquet X, Staels B, O'Connor SE, et al. The anti-obesity effect of rimonabant is associated with an improved serum lipid profile. Diabetes Obes Metab 2005; 7: 65-72.

27. Cota D, Marsicano G, Tschop M, Grubler Y, Flachskamm $\mathrm{C}$, Schubert M, et al. The endogenous cannabinoid system affects energy balance via central orexigenic drive and peripheral lipogenesis. J Clin Invest 2003; 112: 423 31.

28. Ravinet Trillou C, Delgorge C, Menet C, Arnone M, Soubrié P. CB1 cannabinoid receptor knockout in mice leads to leanness, resistance to diet-induced obesity and enhanced leptin sensitivity. Int J Obes Relat Metab Disord 2004; $28:$ 640-8.

29. Fruebis J, Tsao TS, Javorschi S, Ebbets-Reed D, Erickson MR, Yen FT, et al. Proteolytic cleavage product of 30$\mathrm{kDa}$ adipocyte complement-related protein increases fatty acid oxidation in muscle and causes weight loss in mice. Proc Natl Acad Sci USA 2001; 98: 2005-10.

30. Yamauchi T, Kamon J, Waki H, Terauchi Y, Kubota N, Hara $\mathrm{K}$, et al. The fat-derived hormone adiponectin reverses insulin resistance associated with both lipoatrophy and obesity. Nat Med 2001; 7: 941-6.

31. Llanos M, Valenzuela C, Aguirre C, Castillo V, Ronco AM. The endocannabinoid CB1 receptor antagonist, SR141716A, Has paradoxical effects on adult body weight and metabolic alterations due to early post-natal stress. An animal model. Pediatr Res 2009; 65: 479.

32. Matias I, Gonthier MP, Orlando P, Martiadis V, De Petrocellis L, Cervino C, et al. Regulation, function and dysregulation of endocannabinoids in models of adipose and -pancreatic cells and in obesity and hyperglycemia. J Clin Endocrinol Metab 2006; 91: 3171-80.

33. Diraison F, Yankah V, Letexier D, Dusserre E, Jones P, Beylot M. Differences in the regulation of adipose tissue and liver lipogenesis by carbohydrates in humans. J Lipid Res 2003; 44: 846-53.

34. Osei-Hyiaman D, Liu J, Zhou L, Godlewski G, HarveyWhite J, Jeong WI, et al. Hepatic CB1 receptor is required for development of diet-induced steatosis, dyslipidemia, and insulin and leptin resistance in mice. J Clin Invest 2008; 118: 3160-9.

35. Kunos G, Osei-Hyiaman D, Bátkai S, Sharkey KA, Makriyannis A. Should peripheral $\mathrm{CB}(1)$ cannabinoid receptors be selectively targeted for therapeutic gain? Trends Pharmacol Sci 2009; 30: 1-7.

36. Picó JP, Fuentes E, Bermúdez-Silva FJ, Javier DíazMolina F, Ripoll C, Rodríguez de Fonseca F, et al. Can- nabinoid receptors regulate $\mathrm{Ca}(2+)$ signals and insulin secretion in pancreatic beta-cell. Cell Calcium 2006; 39: 155-62.

37. Cavuoto P, McAinch AJ, Hatzinikolas G, Janovská A, Game P, Wittert GA. The expression of receptors for endocannabinoids in human and rodent skeletal muscle. Biochem Biophys Res Commun 2007; 364: 105-10.

38. Pagotto U, Marsicano G, Cota D, Lutz B, Pasquali R. The emerging role of the endocannabinoid system in endocrine regulation and energy balance. Endocr Rev 2006; 27: 73-100.

39. Addy C, Wright H, Van Laere K, Gantz I, Erondu N, Musser BJ, et al. The acyclic CB1R inverse agonist taranabant mediates weight loss by increasing energy expenditure and decreasing caloric intake. Cell Metab 2008; 7: 68-78.

40. Monteleone P, Matías I, Martiadis V, De Petrocellis L, Maj M, Di Marzo V. Blood levels of the endocannabinoid anandamide are increased in anorexia nervosa and in binge-eating disorder, but not in bulimia nervosa. Neuropsychopharmacology 2005; 30: 1216-21.

41. Blüher M, Engeli S, Klöting N, Berndt J, Fasshauer M, Bátkai S, et al. Dysregulation of the peripheral and adipose tissue endocannabinoid system in human abdominal obesity. Diabetes 2006; 55: 3053-60.

42. Côté M, Matías I, Lemieux I, Petrosino S, Alméras N, Després JP, et al. Circulating endocannabinoid levels, abdominal adiposity and related cardiometabolic risk factors in obese men. Int J Obes (Lond) 2007; 31: 692-9.

43. Löfgren P, Sjölin E, Wåhlen K, Hoffstedt J. Human adipose tissue cannabinoid receptor 1 gene expression is not related to fat cell function or adiponectin level. J Clin Endocrinol Metab 2007; 92: 1555-9.

44. Sipe JC, Waalen J, Gerber A, Beutler E. Overweight and obesity associated with a missense polymorphism in fatty acid amide hydrolase (FAAH). Int J Obes 2005; 29: 755-9.

45. Kempf K, Hector J, Strate T, Schwarzloh B, Rose B, Herder $\mathrm{C}$, et al. Immune-mediated activation of the endocannabinoid system in visceral adipose tissue in obesity. Horm Metab Res 2007; 39: 596-600.

46. Pagano C, Pilon C, Calcagno A, Urbanet R, Rossato $M$, Milan $G$, et al. The endogenous cannabinoid system stimulates glucose uptake in human fat cells via phosphatidylinositol 3-kinase and calcium-dependent mechanisms. J Clin Endocrinol Metab 2007; 92: 4810-9.

47. Gazzerro P, Caruso MG, Notarnicola M, Misciagna G, Guerra V, Laezza C, et al. Association between cannabinoid type-1 receptor polymorphism and body mass index in a southern Italian population. Int J Obes (Lond) 2007; 31: 908-12. 
Participación del sistema endocanabinoide en el desarrollo de obesidad - C. Valenzuela et al

48. Peeters A, Beckers S, Mertens I, Van Hul W, Van Gaal L. The G1422A variant of the cannabinoid receptor gene (CNR1) is associated with abdominal adiposity in obese men. Endocrine 2007; 31: 138-41.

49. Enriori PJ, Evans AE, Sinnayah P, Cowley MA. Leptin resistance and obesity. Obesity (Silver Spring) 2006; 14 Suppl 5: 254S-8S.

50. Kalra SP. Central leptin insufficiency syndrome: an interactive etiology for obesity, metabolic and neural diseases and for designing new therapeutic interventions. Peptides 2008; 29: 127-38.

51. Murdolo G, Kempf K, Hammarstedt A, Herder C, Smith $\mathrm{U}$, Jansson PA. Insulin differentially modulates the peripheral endocannabinoid system in human subcutaneous abdominal adipose tissue from lean and obese individuals. J Endocrinol Invest 2007; 30: RC17-21.

52. Després JP, Golay A, Sjöström L; Rimonabant in Obesity-Lipids Study Group. Effects on metabolic risk factors in overweight patients with dyslipidemia. N Engl J Med 2005; 353: 2121-34.

53. Pi-Sunyer FX, Aronne LJ, Heshmati HM, Devin J, Rosenstock J and RIO North America Study Group. Effect of rimonabant, a cannabinoid-1 receptor blocker, on weight and cardiometabolic risk factors in overweight or obese patients. RIO-North America: a randomized controlled trial. JAMA 2006; 295: 761-75.
54. Van Gaal LF, Pi-Sunyer X, Despre's JP, Mc Carthy C, Scheen AJ. Efficacy and safety of rimonabant for improvement of multiple cardiometabolic risk factors in overweight/obese patients: pooled 1-year data from the RIO program. Diabetes Care 2008; 31 (Suppl 2): S22940.

55. Malcher-Lopes R, Di S, Marcheselli VS, Weng FJ, Stuart CT, Bazan NG, et al. Opposing crosstalk between leptin and glucocorticoids rapidly modulates synaptic excitation via endocannabinoid release. J Neurosci 2006; 26 : 6643-50.

56. Malcher-Lopes R, Franco A, Tasker JG. Glucocorticoids shift arachidonic acid metabolism toward endocannabinoid synthesis: a non-genomic anti-inflammatory switch. Eur J Pharmacol 2008; 583: 322-39.

57. Jones D. End of the line for cannabinoid receptor 1 as an anti-obesity target? Nat Rev Drug Discov 2008; 7: 961-2.

58. Nogueiras R, Veyrat-Durebex C, Suchanek PM, Klein M, Tschöp J, Caldwell C, et al. Peripheral, but not central, CB1 antagonism provides food intake-independent metabolic benefits in diet-induced obese rats. Diabetes 2008; 57: 2977-91.

59. McElroy J, Sieracki K, Chorvat R. Non-brain penetrant $\mathrm{CB} 1$ receptor antagonist as novel treatment of obesity and related metabolic disorders. Obesity 2008; 16 (Suppl 1): $S 47$. 